NOTICE: this is the author's version of a work that was accepted for publication in Applied Surface Science. Changes resulting from the publishing process, such as peer review, editing, corrections, structural formatting, and other quality control mechanisms may not be reflected in this document. Changes may have been made to this work since it was submitted for publication. A definitive version was subsequently published in APPLIED SURFACE SCIENCE, [VOL336, (May 2015)] DOI 10.1016/j.apsusc.2014.09.172

\title{
Laser Induced Forward Transfer for front contact improvement in silicon heterojunction solar cells
}

\author{
M. Colina*, A. Morales-Vilches, C. Voz, I. Martín, P. Ortega, A. Orpella, G. López, R. Alcubilla \\ Departament d'Enginyeria Electrònica, Universitat Politècnica de Catalunya, C/ Jordi Girona 1-3, Mòdul C4, 08034 Barcelona, Spain
}

\section{A R T I C L E I N F O}

\section{Article history:}

Received 26 June 2014

Received in revised form

28 September 2014

Accepted 29 September 2014

Available online $\mathrm{xxx}$

\section{Keywords:}

LIFT

Silicon heterojunction solar cell

Laser direct-write

\begin{abstract}
A B S T R A C T
In this work the Laser Induced Forward Transfer (LIFT) technique is investigated to create n-doped regions on p-type c-Si substrates. The precursor source of LIFT consisted in a phosphorous-doped hydrogenated amorphous silicon layer grown by Plasma Enhanced Chemical Vapor Deposition (PECVD) onto a transparent substrate. Transfer of the doping atoms occurs when a sequence of laser pulses impinging onto the doped layer propels the material toward the substrate. The laser irradiation not only transfers the doping material but also produces a local heating that promotes its diffusion into the substrate. The laser employed was a $1064 \mathrm{~nm}$, lamp-pumped system, working at pulse durations of 100 and $400 \mathrm{~ns}$. In order to obtain a good electrical performance a comprehensive optimization of the applied laser fluency and number of pulses was carried out. Subsequently, arrays of $n+p$ local junctions were created by LIFT and the resulting $J-V$ curves demonstrated the formation of good quality $n+$ regions. These structures were finally incorporated to enhance the front contact in conventional silicon heterojunction solar cells leading to an improvement of conversion efficiency.
\end{abstract}

(c) 2014 Elsevier B.V. All rights reserved.

\section{Introduction}

Potential applications of Laser Induced Forward Transfer (LIFT) are being extensively explored by the scientific community in the last years [1,2]. In this period, theoretical and practical analyses have contributed to the understanding of the physical phenomena behind the LIFT process [3]. Additionally, more fields of applications are being proposed for deposition of materials via LIFT. In particular, photovoltaic technology seems to be a potential candidate that might take advantage of the LIFT technology for the deposition of a large variety of materials of both inorganic and organic nature [4-6]. In general, direct write (DW) techniques exhibit the advantage of avoiding the use of photolithography steps and of serigraphy masks, thus making these techniques a suitable and flexible alternative when different types of patterns and structures need to be defined [7]. Thus, being LIFT among said DW techniques, it can take advantage not only of this previous described property but also of the variety of substrates that can be used when selecting the right laser characteristics (especially, wavelength and pulse duration).

Among solar cells based on silicon technology, heterojunction (HT) cells present a potential solution to reduce the expenses

\footnotetext{
* Corresponding author. Tel.: +34 9340174 88; fax: +34 934016756.

E-mail addresses: monicacolinb@gmail.com, dsolerv@gmail.com (M. Colina).
}

associated with the use of relatively thick silicon wafers. Indeed, the amorphous silicon layers in HT cells can be deposited in thinner wafers which could result in a significant decrease of the amount of crystalline silicon to be employed in the final device. In this scenario, the most remarkable category of HT solar cells with already demonstrated higher efficiency corresponds to the HIT (Heterojunction with Intrinsic Thin-film) technology. In 2009, the HIT structure showed its potential with a $22.8 \%$ efficiency even when using a $98 \mu \mathrm{m}$ thick silicon wafer. This result is in fact comparable to the $23 \%$ efficiency exhibited by HIT cells made on silicon wafers with a $200 \mu \mathrm{m}$ thickness [8]. Recently, a HIT device exhibiting a record efficiency of $24.7 \%$ and manufactured with a silicon wafer of industrial size and a thickness of $98 \mu \mathrm{m}$ has been reported [9]. Based on these successful results, the interest to investigate more in the preparation of more efficient $\mathrm{HJ}$ solar cells is obvious.

By joining the importance to get improvements in the development of $\mathrm{HJ}$ solar cells and the increasing application of LIFT technology in photovoltaics [6,10-14], this work presents the first application of LIFT for the improvement of the front contact series resistance of silicon $\mathrm{HJ}$ solar cells. In first place, the material acting as a doping precursor and the experimental assembly will be presented. Next, the steps implemented for the fabrication of the silicon HJ solar cell will be described. Afterwards, laser parameter optimization for the material transfer process will be shown, and finally, the electrical performance of the fabricated solar cells will be presented and analyzed. 


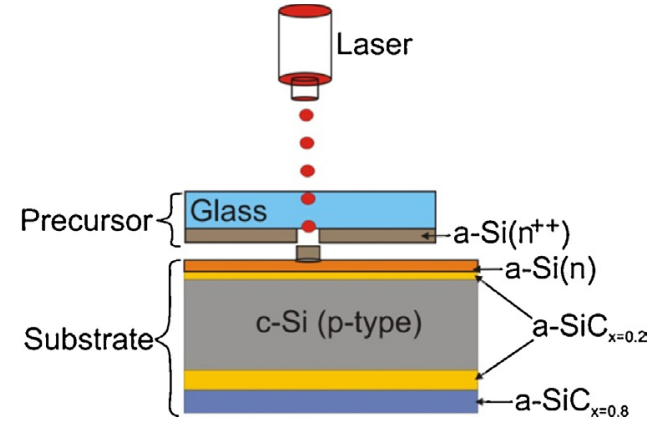

Fig. 1. Diagram of the LIFT technique when using a precursor of a-Si:H $\left(n^{++}\right)$for the fabrication of $\mathrm{HJ}$ solar cell structures.

\section{Materials and methods}

A $100 \mathrm{~nm}$ hydrogenated amorphous silicon film with a high phosphorus content, a-Si:H $\left(\mathrm{n}^{++}\right)$, grown onto a glass substrate was used as dopant precursor in the LIFT process. This layer was deposited by means of a PECVD system $(13.56 \mathrm{MHz})$ from Elettrorava S.p.A. The laser employed for all the experiments was a lamp pumped Nd:YAG (StarMark SMP 100II Rofin-Baassel) Gaussian beam operating at $1064 \mathrm{~nm}$ and $4 \mathrm{kHz}$ repetition frequency. In all cases, the transfer process consisted in a finite number of pulses impinging onto a system comprising a precursor and a substrate, as depicted in Fig. 1. Although the precursor and the substrate are presented in Fig. 1 with a gap between them for illustrative purposes, both parts of the system were in fact put together to optimize the laser transfer process.

Two fabrication procedures were employed according to two different purposes: (1) diode fabrication and (2) HJ solar cell fabrication. The silicon substrate used for the experiments consisted in $\langle 100\rangle$ p-type FZ c-Si wafers with a thickness of $280 \mu \mathrm{m}$ and a resistivity of $2.5 \Omega \mathrm{cm}$. These wafers were cleaned by means of a complete RCA sequence previous to the deposition of the different layers.

For the diode fabrication experiment, a stack consisting of a hydrogenated amorphous silicon carbide, a- $\mathrm{SiC}_{0.2}: \mathrm{H}$, passivation layer $(\sim 3 \mathrm{~nm})$ and a phosphorus doped hydrogenated amorphous silicon, a-Si:H (n), emitter layer were deposited on the front side of the wafer. Likewise, a stack of a-SiC $\mathrm{S}_{0.2}: \mathrm{H}$ film for surface passivation and $\mathrm{a}-\mathrm{SiC}_{0.8}: \mathrm{H}$ film for improving back reflector properties was also deposited by PECVD on the rear side of the silicon wafer. More detailed information regarding PECVD fabrication parameters of similar layers can be found in previous works [15,16]. After the PECVD depositions, the following steps were carried out for the diode experiment:

(1) Phosphorous Laser Transfer

(2) Front transparent contact deposition (indium tin oxide, ITO, by means of sputtering)

(3) Photolithography (to define the diode areas by means of a photoresist)

(4) Emitter dry etching (tetrafluoromethane, $\mathrm{CF}_{4}$, PECVD room temperature etching)

(5) HF $1 \%$ bath for removal of ITO defined areas

(6) Photoresist removal

(7) Rear metal deposition by thermal evaporation

(8) Rear contact formation by laser firing contact, LFC, according to the procedure shown elsewhere [17]

(9) Front metal deposition by thermal evaporation.

Different laser parameters such as pulse energy, number of pulses and pulse duration were varied for the fabrication of the

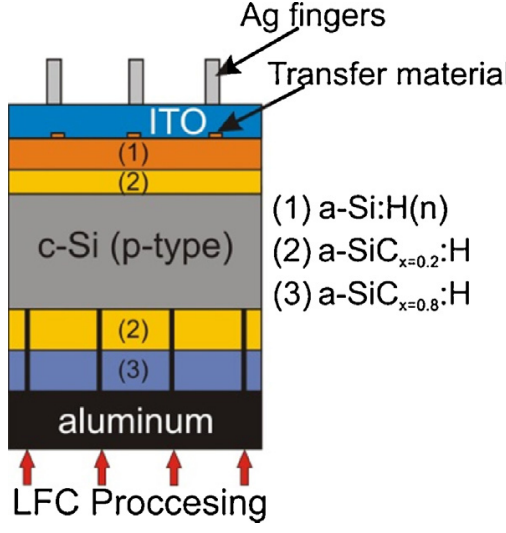

Fig. 2. Structure of the crystalline silicon HJ solar cell treated by LIFT where it is illustrated that the doped material is transferred exactly underneath the Ag fingers.

different diodes. Each diode had a $5 \mathrm{~cm}^{2}$ area in which an array of $15 \times 13$ laser spots with a $300 \mu \mathrm{m}$ pitch was transferred in order to enhance the series resistance $\left(R_{\mathrm{s}}\right)$ of the diodes. In order to identify the laser parameters leading to an optimal series resistance, $J-V$ measurements were carried out for each fabricated diode.

Once the laser parameters leading to a series resistance improvement were identified, a set of $8 \mathrm{HJ}$ solar cells of $1 \mathrm{~cm}^{2}$ were fabricated while applying LIFT onto the emitter side of the cells. A set of 8 control cells, without LIFT treatment, was also fabricated in the same wafer. The procedure employed for the fabrication of these equivalent control p-type $\mathrm{HJ}$ cells is described in a previous work [18]. It is important to mention that in those cells where LIFT was applied, laser transferred spots were arranged exactly under the front contact silver fingers as shown in Fig. 2. Finally, dark and illumination (AM 1.5) J-V measurements were carried out both for the LIFT and control cells.

\section{Results}

\subsection{Morphology results}

First, a morphological evaluation of the spots resulting from the LIFT process was carried out. Fig. 3 shows optical microscopy images of the resulting spots performed at different laser pulse energies. Each spot is the result of 13 pulses of 400 ns duration and $4 \mathrm{kHz}$ frequency impinging onto the system formed by the precursor and the substrate. As the energy increases, the spot diameter also increases. Clear evidences of surface modification are found even when the lowest value of energy is evaluated. Material splashing at the nanometric scale, likely arising from the doping precursor layer, can be observed for the three lower energy values. This splash is distributed in a circular area where the a-Si (n) was possibly affected. Finally, the silicon base seems to have been melted and re-solidified when analyzing the result obtained with the highest laser energy value. A similar morphological analysis was made when varying the number of pulses at a same energy. In this case, the morphology of the spots did not exhibit significant variations between them (less than $2 \%$ ), at least for the explored range $(3,6$, 13 and 20).

The material ablation threshold when working with Gaussian beams can be determined applying the method disclosed by Liu [19]. Once the ablation threshold is reached, material alteration begins to be optically evident. Thus, the ablation fluence $F_{a}$ is defined by the following equation:

$F_{a}=\frac{2 E}{\pi \omega^{2}} e \frac{-2 r^{2}}{\omega^{2}}$ 

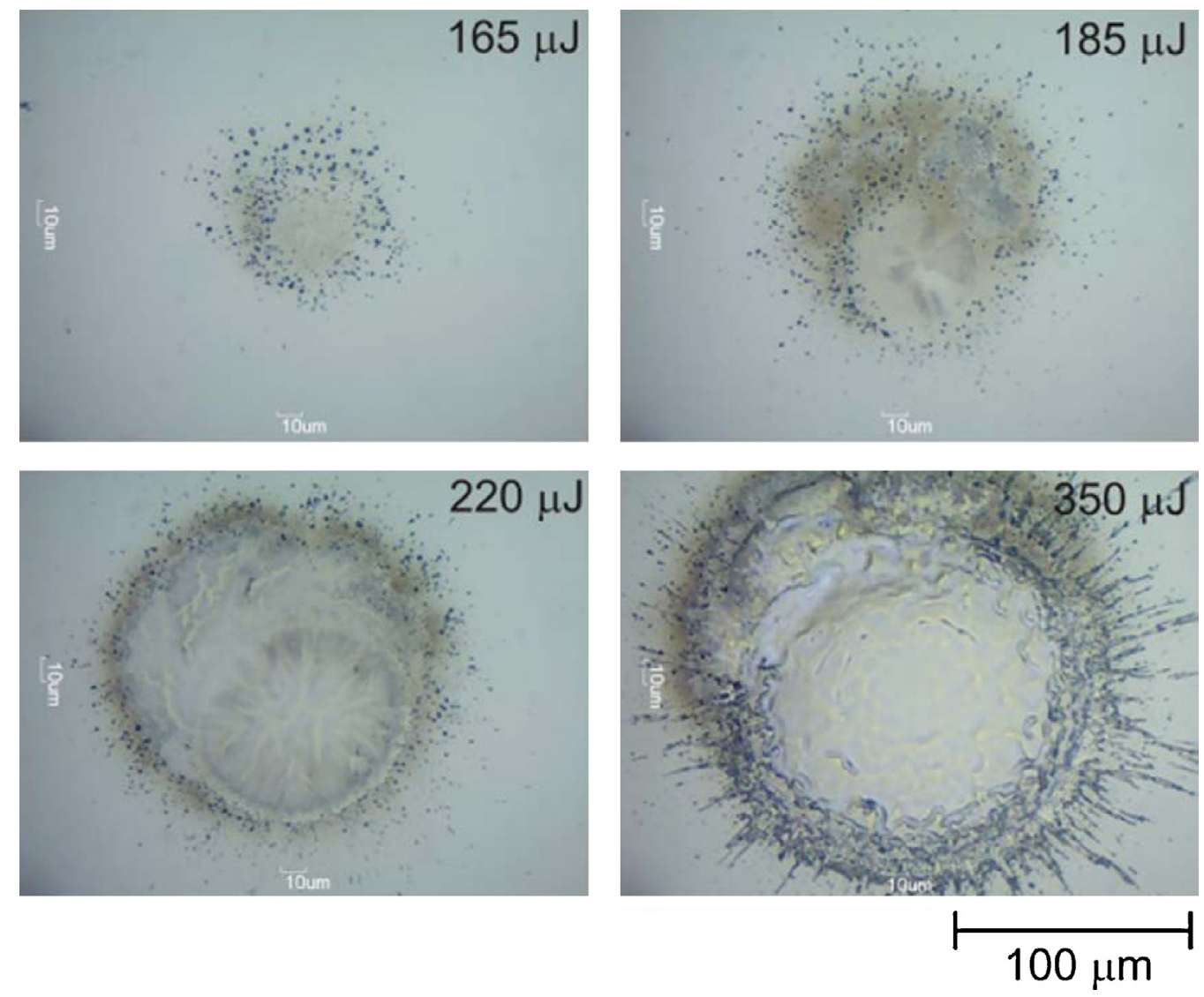

Fig. 3. Morphological results as a function of laser energy variation when applying LIFT onto a Silicon HJ structure.

where $E$ is the pulse energy, $\omega$ is the beam radius and $r$ is the spot radius.

The measurement of the spot radius at different pulse energies allowed the determination of the ablation threshold when putting the precursor doping layer in the beam path before the substrate. Fig. 4(a) shows the results of the measured spot radius as a function of the laser pulse energy. A lineal fitting of the experimental data allows extracting the ablation threshold fluence, which results in about $2.15 \mathrm{~J} / \mathrm{cm}^{2}$. Fig. 4(b) shows two Gaussian curves, one corresponding to a peak that does not reach the threshold $(E=130 \mu \mathrm{J})$ and the other one corresponding to an energy peak of $185 \mu \mathrm{J}$ (above the threshold). In this second Gaussian, the diameter, as determined by the interception with the ablation threshold, is of about $60 \mu \mathrm{m}$. This diameter coincides with the circular area defined onto the substrate when the splashing is not taken into account, as it is demonstrated by the optical image insets in Fig. 4(b). In the following paragraphs, it will be seen that this particular energy interestingly corresponds to the optimal electrical behavior.

\subsection{Electrical results of the $n+/ p$ diodes}

$J-V$ characterization was carried out for the different arrays created by LIFT. It is important to clarify that there is no need to create an array for the creation of a diode since the already present emitter layer is the element responsible for the creation of the $p-n$ junction. Nevertheless, the typical reduction on the current injected at high injection voltage levels, which is attributed to the presence of series resistances, is less marked for those diodes where LIFT was applied. This fact, which can be assessed when observing the curves presented in Fig. 5, indicates that the LIFT process transfers phosphorous dopant atoms toward the substrate. These atoms remain into the substrate and positively affect the performance of the diode in the high voltage regime.

The laser pulse energy was varied for each LIFT device to correlate the energy with the electrical behavior. In addition, other optimization studies were performed by varying the number of pulses, the repetition frequency and the pulse duration. The studies of frequency and pulse duration will be omitted in this document as the laser energy and the number of pulses are the parameters that clearly had the major influence on the electrical performance of the devices. The $J-V$ measurements carried out for the control and the LIFT devices are presented in Fig. 5. All the devices had an exponential behavior with six order of magnitude of rectification. A first interesting aspect is found from $0.6 \mathrm{~V}$, where higher levels of current density are distinguished for those diodes with LIFT. This fact is a consequence of an improvement of the series resistance in comparison with that diode (labeled as "emitter" in the graphs) without LIFT treatment. Fig. 5(a) shows the $J-V$ curves at different laser energies and a fixed number of pulses. It is also observed that for those diodes with a stronger LIFT treatment (that is, the two highest energies), the $\mathrm{p}-\mathrm{n}$ junction results completely damaged. For these particular energies, the obtained behavior is close to a typical metal-semiconductor diode. This fact indicates a total lack of phosphorous doped atoms, which were possibly ablated by the laser treatment itself. For intermediate energy values, an anomaly appears on the curves in the low injection regime. This is presumably due to certain damage in the emitter layer. Finally, for the lowest pulse energy values, the $J-V$ curves are quite similar to the control (emitter) result. Overall, $185 \mu \mathrm{J}$ resulting in the optimum energy and gave place to the best electrical results. Although the influence of the number of pulses is less marked than the influence of the laser energy as demonstrated in Fig. 5(b), it can be seen that the anomalies at the low injection regime seem to be more evident 
(a) 24

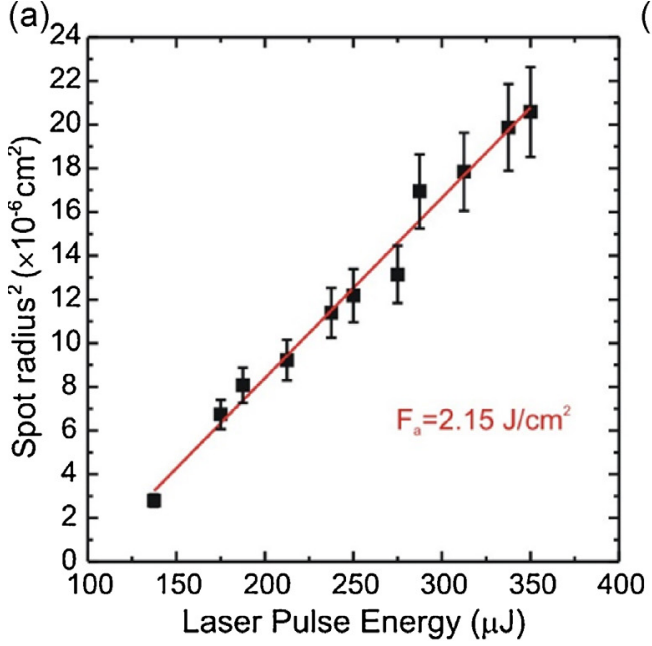

(b)

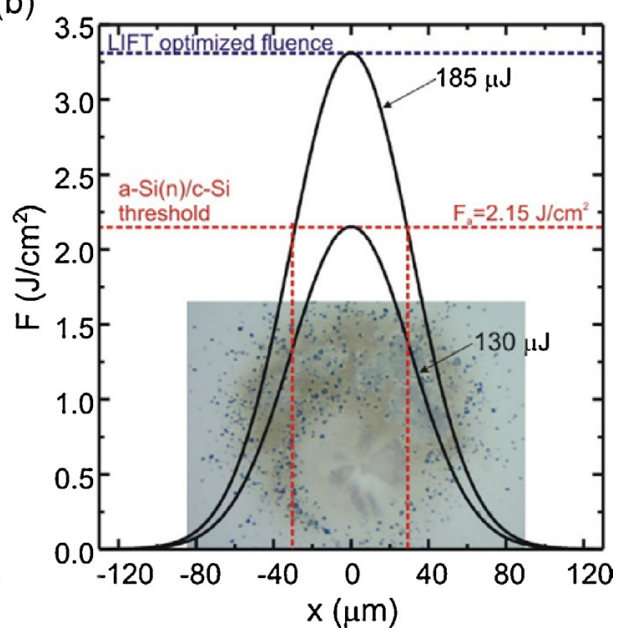

Fig. 4. Ablation threshold study: (a) laser spot size as a function of the pulse energy and (b) Energy distribution of two characteristic Gaussian pulses.

as the number of pulses increases. Nevertheless, this behavior does not excessively affect the performance of the device in the region of $0.5-0.7 \mathrm{~V}$. Thus, a number of pulses of 13 with an optimized energy were selected to carry out the application of LIFT on the HJ solar cells.

For those energy values where a clear $\mathrm{p}-\mathrm{n}$ junction behavior was maintained after the LIFT treatment, the value of $R_{\mathrm{S}}$ resulted in around $0.6 \Omega \mathrm{cm}^{2}$ deduced from the $J-V$ values at very high voltages. As far as the emitter layer with no LIFT is concerned, the calculated $R_{\mathrm{S}}$ value was around $1.2 \Omega \mathrm{cm}^{2}$. Therefore, on the basis of these experimental evidences, it can be stated that the use of LIFT for the reduction of the series resistance in a-Si HJ diodes has been demonstrated.

\section{3. a-Si HJ cells electrical results}

Once the diode experiments shown in Section 3.2 allowed the optimization of the laser parameters, a series of p-type $1 \mathrm{~cm}^{2} \mathrm{a}-\mathrm{Si}$ $\mathrm{HJ}$ solar cells was prepared applying the LIFT process. The $\mathrm{n}^{++}$ transferred spots were placed under the silver fingers of the front contact of the cells (see Fig. 2) and a $300 \mu \mathrm{m}$ pitch was maintained between the spots. Some control devices were also fabricated and measured for comparative purposes. The laser parameters corresponding to $185 \mu \mathrm{J}$ pulse energy, $4 \mathrm{kHz}$ repetition frequency and 13 pulses per spot were used for the LIFT cells according to the best diode results shown in the previous section. The electrical performance of the fabricated cells was measured both under dark and illuminated conditions.

A representative example of the $J-V$ dark curves obtained for LIFT and NO-LIFT cells is presented in Fig. 6. Here, two main features must be remarked: (1) the lower series resistance obtained for the LIFT cell as determined from the analysis in the high injection region and (2) the existence of a low injection mechanism that increases the current in the range from 0.0 to $0.5 \mathrm{~V}$ in comparison to the NOLIFT cell. The first point confirmed that LIFT is a promising and real alternative to improve the series resistance of the front contact of silicon-based $\mathrm{HJ}$ solar cells without using any additional thermal or photolithography step. The second point might be related to an undesirable damage probably created in the emitter layer, being a consequence of the local heating generated by the laser pulses.

A fitting to a two diode model according to the following expression was applied to the experimentally obtained curves:

$J=J_{01} \exp \left(\frac{V}{V_{t}}\right)+J_{02} \exp \left(\frac{V}{2 V_{t}}\right)$

(b)

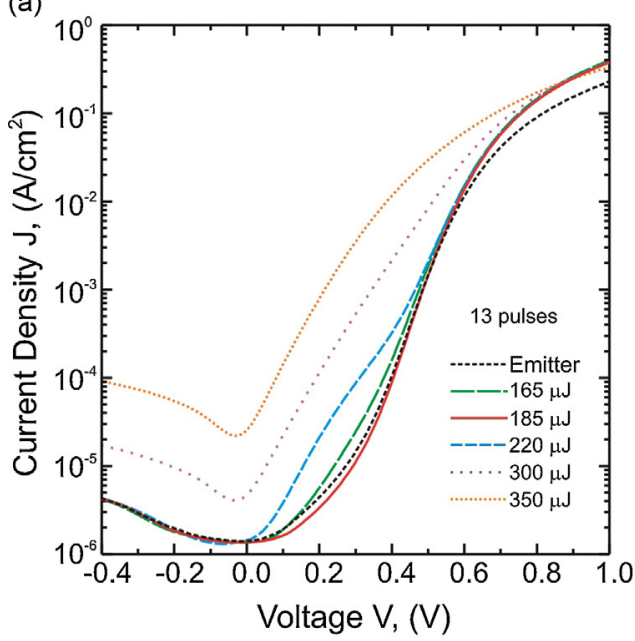

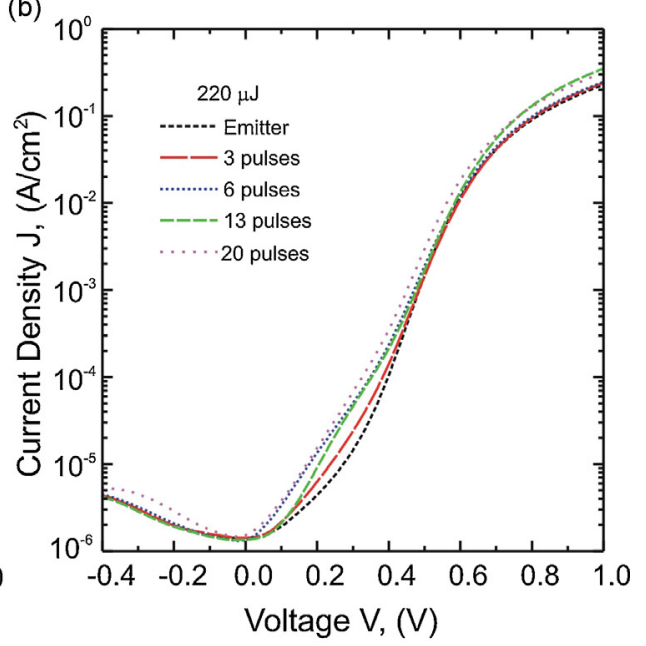

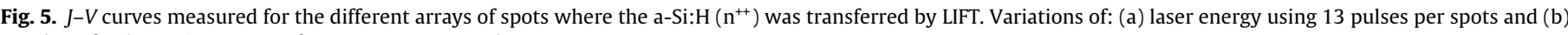
number of pulses using $220 \mu \mathrm{J}$ of energy are presented. 


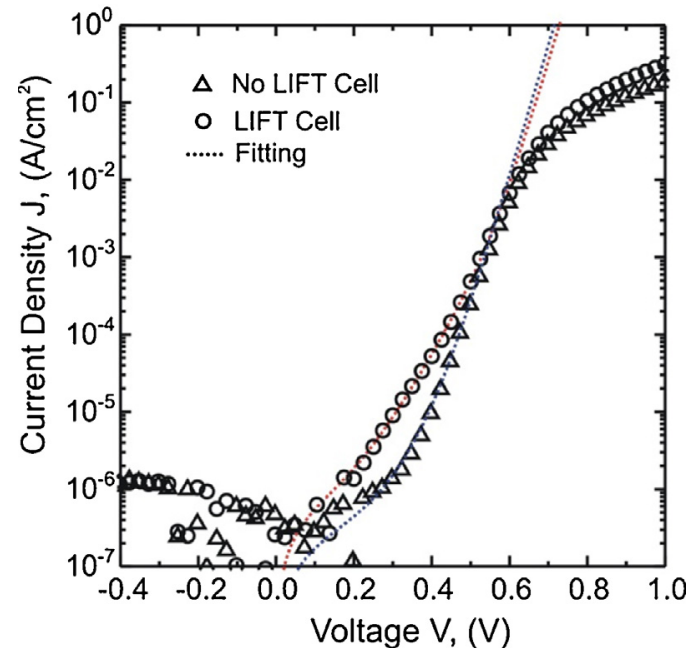

Fig. 6. Dark $J-V$ curves corresponding to two c-Si HJ solar cells with and without phosphorus spots transferred by means of LIFT.

where $V$ is the voltage, $V_{t}$ is the thermal potential, $J_{01}$ is the diffusion current density and $J_{02}$ is the recombination current density.

The parameters extracted from the fitting of all the fabricated cells are statistically represented in Fig. 7. Again, the series resistance is lower for the LIFT cells which confirms the viability of a-Si $\left(\mathrm{n}^{++}\right)$LIFT for the improvement of the front contact. In addition, the diffusion mechanisms, represented by the $J_{01}$ values, are comparable in both types of cells. Finally, the recombination mechanisms, represented by $J_{02}$, results higher for the LIFT cells, which can be indicative of some current leaks induced by the laser treatment.

The photovoltaic parameters obtained under illuminated conditions for all the fabricated $\mathrm{HJ}$ solar cells are shown in Fig. 8. In order to compare the electrical performance of the cells with and without LIFT a statistical analysis was carried out. All the graphs presented in Fig. 8 show the maximum, the minimum, the mean and the average value for each process and each electrical parameter.

First of all, it has to be remarked that all the photovoltaic parameters are in the same range of values when comparing the results obtained for the cells with and without LIFT. In second place, the lower open circuit voltage, $V_{o c}$, exhibited by the LIFT cells could indicate again a slight damage created on the emitter layer due to the laser process. This behavior was already expected from the analysis of the dark measurements, where the recombination current was higher for those cells with LIFT treatment. The incorporation of damages in either the silicon crystal or the amorphous emitter may be responsible for the creation of recombination mechanisms that negatively affect the cell performance. On the contrary, the short circuit current density, $J_{s c}$, values are higher for the LIFT cells than for the control ones. This means that LIFT cells can extract more current from the photovoltaic process.

Finally, similar fill factor (FF) values are obtained for both type of devices. In this case, the decrease in $R_{\mathrm{S}}$ occurs together with the appearance of a non-ideal current mechanism at low voltages. Then, the expected positive effect in FF by $R_{\mathrm{S}}$ reduction is compensated by the non-ideal exponential trend. Nevertheless, higher efficiencies were achieved with LIFT process demonstrating that the LIFT technique is a very promising alternative to improve the front contact of a-Si HJ solar cells. There is still room to improve the laser parameterization and, in particular, less aggressive wavelengths and pulse durations can be tested in order to reduce the damage in the emitter film and, consequently, to reduce the recombination mechanisms that seem to be limiting the performance of the solar cells presented in this work.

\section{Discussion}

Based on the morphology results, it was already anticipated that the laser employed for this study might probably affect the properties of the very thin $(\sim 12 \mathrm{~nm})$ emitter layer of the silicon $\mathrm{HJ}$ solar cell. In all cases, from the energy point of view, this layer resulted affected to some variable extend. Nevertheless, this work did not intent to demonstrate that all the laser parameters selected are suitable for the successful application of LIFT. On the contrary, what this work shows is the viability of applying LIFT to improve the front contact resistance, and even more, the potential application of LIFT in other types of solar cells with front contact issues and where the substrate should be kept unmodified. Thus, the versatility of LIFT must be remarked when looking at both the substrates and the materials that can be employed to achieve the transfer. In addition, the fact that the laser energy threshold allows in some way the prediction of an optimal fluence value (Fig. $4 \mathrm{~b}$ ) is very attractive in order to stablish systematic procedures to use LIFT for this kind of application.

According to the results obtained for the $\mathrm{HJ}$ cells measured under dark conditions, two different effects were observed when
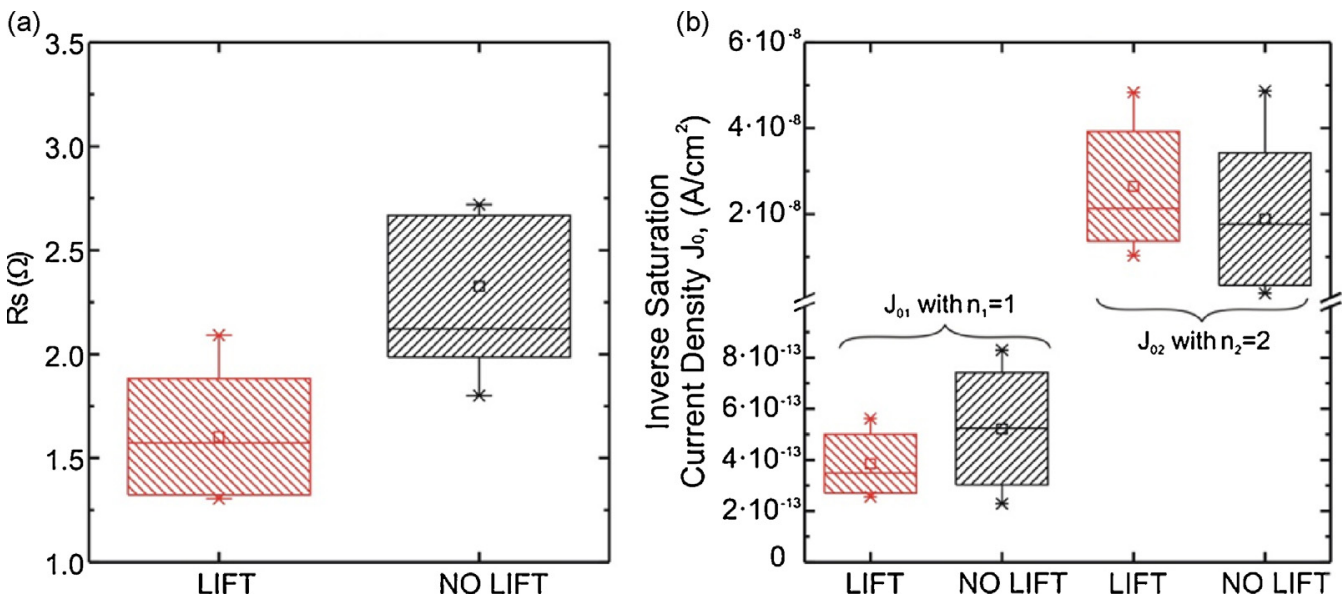

Fig. 7. Results of (a) $R_{\mathrm{s}}$ and (b) $J_{0}$ extracted from the two-diodes fitting applied to all the HJ solar cells fabricated with and without LIFT. 

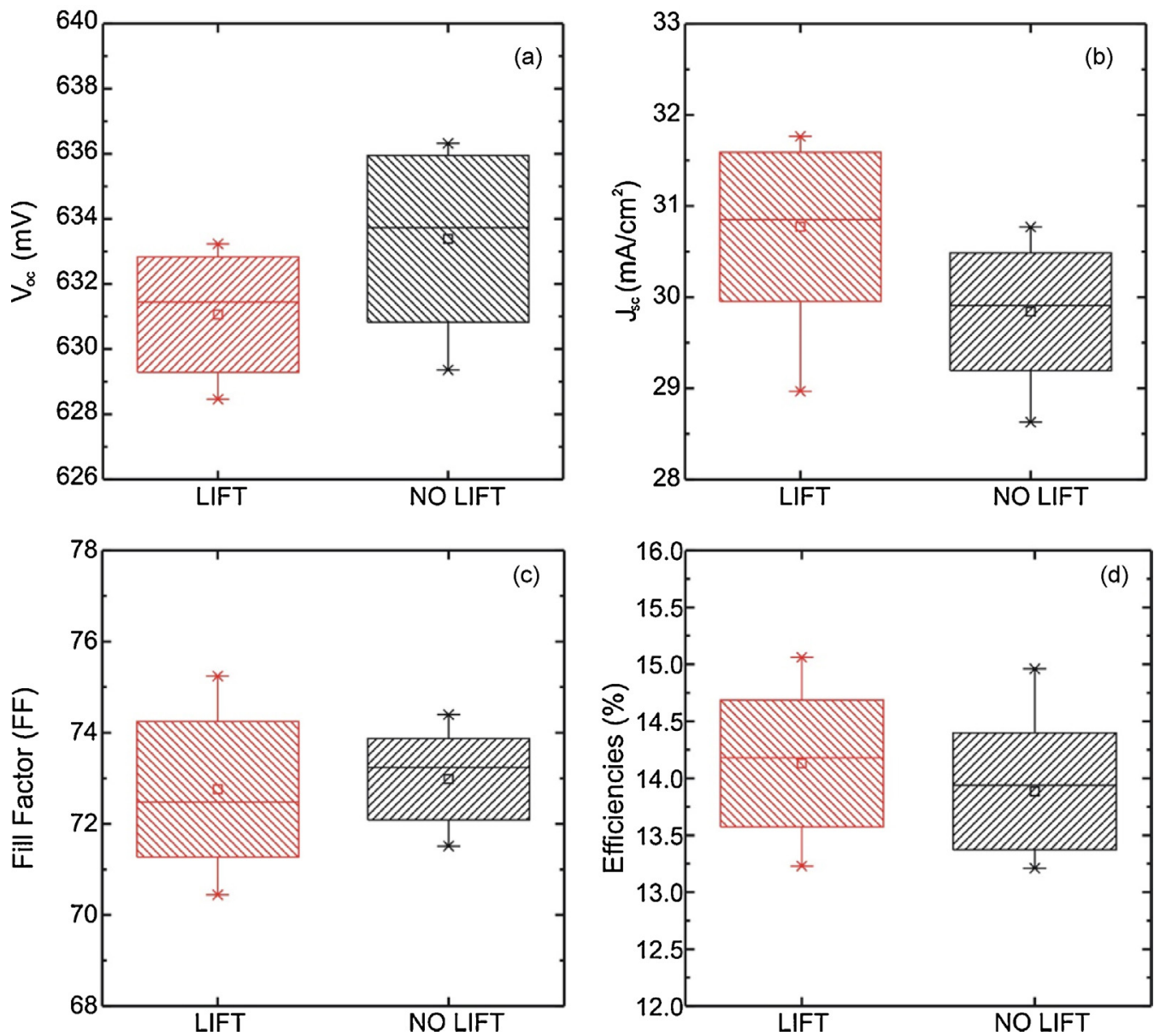

Fig. 8. Statistical results of the photovoltaic parameters for all the fabricated solar cells with and without a-Si:H ( $\left.\mathrm{n}^{++}\right)$transferred by means of LIFT.

using the LIFT strategy. The first and more important when taking into account the scope of this work, was the improvement obtained in the series resistance of the cell. The second effect corresponded to the appearance of a non-common low injection mechanism that could degrade the performance of the cell. A local heating of the emitter layer can be proposed as a plausible reason for the latter effect, this being probably consequence of using a laser wavelength $(1064 \mathrm{~nm})$ and a pulse duration $(400 \mathrm{~ns})$ that are more prone to thermal interactions. In that sense, the statistical analysis presented for all the $\mathrm{HJ}$ cells pointed to an increase of the recombination mechanism when applying LIFT, this being an indicator of certain damage induced in either the Si crystal or the emitter layer. One possibility to perform the LIFT process without inducing damage on the emitter and on the silicon base could be the use of laser wavelengths for which these materials have lower optical absorption lengths. There are already some experiences reporting the use of different wavelengths for laser doping of c-Si [20]. Thus, further studies involving assays with VIS and UV lasers might be interesting in order to optimize this LIFT application.

Last but not least, the analysis of the electrical performance of the cells under illumination confirmed the viability of LIFT for the improvement of the front contact resistance. Despite the fact that open circuit voltages were apparently affected by the laser treatment, it has to be noted that this effect was compensated by the positive effects and the overall efficiency of the devices was higher than when not using LIFT. In that sense, it is worth mentioning that the adequate selection of the laser parameters opens a wide range of promising applications for this technique in the achievement of better device performance.

\section{Conclusion}

The use of LIFT for the transfer of phosphorous doped material by means of a $1064 \mathrm{~nm}, 400 \mathrm{~ns}$ laser was demonstrated in this work. Amorphous silicon doped layers were an adequate precursor for the LIFT process application. In particular, the application of LIFT for the transfer of phosphorous atoms leads to a reduction of the series resistance of a-Si HJ diodes. In addition, a statistical comparison between complete a-Si HJ cells with and without LIFT was also performed. The obtained results demonstrated the viability of LIFT for the improvement of the front contact resistance in a-Si $\mathrm{HJ}$ solar cells.

In summary, cell results confirmed that LIFT is a real alternative to improve the series resistance of the front contact of a silicon-based $\mathrm{HJ}$ solar cell without using any additional thermal or photolithography steps and, eventually, a real alternative to improve the efficiency and competitiveness and photovoltaic devices.

\section{Acknowledgments}

This work has been funded by Spanish government through project TEC2011-26329 and it has also received funding from the European Union's Seventh Framework Programme for research, 
technological development and demonstration under Grant Agreement No. 608498.

\section{References}

[1] A. Pique, H. Kim, A.B. Craig, Laser forward transfer of electronic and power generating material, in: C. Phipps (Ed.), Laser Ablation and Its Applications, Springer, 2007, pp. 339-373.

[2] S.A. Mathews, R.C.Y. Auyeung, A. Piqué, Use of laser direct-write in microelectronics assembly, J. Laser Micro/Nanoeng. 2 (2007) 103-107.

[3] T.C. Ro?der, J.R. Ko?hler, Physical model for the laser induced forward transfer process, Appl. Phys. Lett. 100 (2012) 071603.

[4] M. Kandyla, S. Chatzandroulis, I. Zergioti, Laser induced forward transfer of conducting polymers, Opto-Electron. Rev. 18 (2010) 345-351.

[5] J. Shaw-Stewart, T. Mattle, T. Lippert, M. Nagel, F. Nüesch, A. Wokaun, The optimisation of the laser-induced forward transfer process for fabrication of polyfluorene-based organic light-emitting diode pixels, Appl. Surf. Sci. 278 (2013) 341-346.

[6] R. Ferré, R. Gogolin, J. Müller, N.-P. Harder, R. Brendel, Laser transfer doping for contacting n-type crystalline Si solar cells, Phys. Status Solidi 208 (2011) 1964-1966.

[7] K.K.B. Hon, L. Li, I.M. Hutchings, Direct writing technology - advances and developments, CIRP Ann. Manuf. Technol. 57 (2008) 601-620.

[8] T.N. Mikio Taguchi, Y. Tsunomura, H. Inoue, S. Taira, E.M. Toshiaki Baba, H. Sakata, High-efficiency hit solar cell on thin $(<100 \mu \mathrm{m})$ silicon wafer, in: 24 th European Photovoltaic Solar Energy Conference, Hamburg, Germany, 2009, pp. 1690-1693.

[9] M. Taguchi, A. Yano, S. Tohoda, K. Matsuyama, Y. Nakamura, T. Nishiwaki, K. Fujita, E. Maruyama, 24.7\% record efficiency HIT solar cell on thin silicon wafer, IEEE J. Photovolt. 4 (2014) 96-99.
[10] E. Hoffmann, T.C. Röder, J.R. Köhler, Self-doping laser transferred contacts for c-Si solar cells, IEEE J. Photovolt. (2011) 1059-1062.

[11] T.C. Röder, E. Hoffmann, B. Konrad, J.R. Köhler, Low temperature laser metallization for silicon solar cells, Energy Proc. 8 (2011) 552-557.

[12] A. Bogaerts, Z. Chen, R. Gijbels, A. Vertes, Laser ablation for analytical sampling: what can we learn from modeling, Spectrochim. Acta Part B: At. Spectrosc. 58 (2003) 1867-1893.

[13] N. Wehkamp, A. Fell, J. Bartsch, F. Granek, Laser chemical metal deposition for silicon solar cell metallization, Energy Proc. 21 (2012) 47-57.

[14] L. Wang, D.E. Carlson, M.C. Gupta, Silicon solar cells based on all-lasertransferred contacts, Prog. Photovolt. Res. Appl. (2013), http://dx.doi.org/ 10.1002/pip.2395.

[15] A. Morales-Vilches, C. Voz, M. Colina, G. López, I. Martín, P. Ortega, A. Orpella, R. Alcubilla, Recovery of indium-tin-oxide/silicon heterojunction solar cells by thermal annealing, Energy Proc. 44 (2014) 3-9.

[16] A. Morales, C. Voz, M. Colina, G. López, I. Martín, A. Orpella, J. Puigdollers, M. García, R. Alcubilla, Progress in silicon heterojunction solar cell fabrication with rear laser-fired contacts, IEE Explor. 22 (2012) 345-348.

[17] P. Ortega, A. Orpella, I. Martín, M. Colina, G. López, C. Voz, M.I. Sánchez, C. Molpeceres, R. Alcubilla, Laser-fired contact optimization in c-Si solar cells, Prog. Photovolt. Res. Appl. 20 (2012) 173-180.

[18] M. Colina, I. Martín, C. Voz, A. Morales-Vilches, P. Ortega, G. López, A. Orpella, M. García-Molina, D. Muñoz-Martín, M.I. Sánchez-Aniorte, C. Molpeceres, R. Alcubilla, Optimization of laser processes for local rear contacting of passivated silicon solar cells, Energy Proc. 44 (May) (2014) 234-243.

[19] J.M. Liu, Simple technique for measurements of pulsed Gaussian-beam spot size, Opt. Lett. 7 (1982) 196-198.

[20] C. Molpeceres, M.I. Sánchez-Aniorte, M. Morales, D. Muñoz, I. Martín, P. Ortega, M. Colina, C. Voz, R. Alcubilla, Influence of wavelength on laser doping and laser firing contact processes for crystal silicon solar cells, Proc. SPIE 8473 (2012) 847308 . 